HEARES 01496

\title{
Laser Doppler velocimetry of basilar membrane vibration *
}

\author{
Alfred L. Nuttall, David F. Dolan and G. Avinash \\ University of Michigan Medical School, Kresge Hearing Research Institute, Ann Arbor, Michigan, U.S.A.
}

(Received 15 May 1990; accepted 5 August 1990)

\begin{abstract}
A method is described for the measurcment of basilar membranc (BM) vibration using a commercially made laser Doppler velocimeter (LDV). The instrumentation was coupled to a compound microscope which served to visualize reflective glass microbeads placed on the BM. The laser beam of the LDV was focused in the microscope object plane and positioned over the reflective bead. We show examples of frequency tuning curves and displacement input/output intensity functions obtained with the technique.
\end{abstract}

Guinea pig; Tuning curves; Laser Doppler vibrometry; Microscopy

\section{Introduction}

The development of the LDV as a commercial instrument offers a new tool for cochlear physiological studies. This instrument is the maturation of the (custom-built) research type laser interferometer into an easy to use and reliable consumer product, albeit an expensive one for the typical biomedical laboratory. The LDV used in the current work is a heterodyne-type interferometer and derives from the class of instruments used for detecting particle flow (laser Doppler anemometry) (Durrani and Greated, 1977). Certain laser based methods have already been used to study basilar membrane motion. They include speckle detection (Kohlloffel 1972a,b), homodyne interferometry (Khanna 1986; Khanna et al., 1986; Khanna and Leonard, 1982), and heterodyne interferometry (Willemin et al., 1988).

The Polytec Corp LDV allows one to make point-by-point measurements of the magnitude

Correspondence to: A.L. Nuttall, University of Michigan Medical School, Kresge Hearing Research Institute, Ann Arbor, MI 48109-0506, U.S.A.

* Presented in part at the Midwinter Meeting Association for Research in Otolaryngology, 1990, St. Petersburg Beach, FL and Society for Neuroscience Meeting 1989, Phoenix, AZ. and phase of the velocity of the basilar membrane by focusing a laser beam from the optical head of the instrument onto the location to be tested. The same optical head collects the light scattered from the moving surface and relays it via an optical fiber, to the signal processor. The LDV can also be used for making differential measurements using two optical heads, the second one carrying the reference beam of the interferometer. The Polytec signal processor has a very fast response: a change in surface velocity from zero to $10 \mathrm{~m} / \mathrm{s}$ (step response) being correctly displaced after only 170 nanoseconds. This kind of response gives the user the capability of measuring transient activity on the membrane.

The back reflected light, which contains all of the vibrational information, is collected by the same optical head and light guide fiber which couples the laser beam to the microscope. The use of a single optical head/fiber system in the Polytec Corp. instrument eliminates the need for alignment of source and receiver elements. A quarterwaveplate in front of the collecting optics separates the outgoing light from the incoming. The back reflected light from the optical head and the reference beam are made to interfere, where the intensity modulation in the interference patterns contains the vibrational information. Specifically, the light scattering off the vibrating membrane 
will have a Doppler shift $f_{D}$, which is linearly dependent on the component of the velocity $v$ of the surface along the direction of the laser light according to the formula: $f_{D}=2 v / \lambda$, where $\lambda$ is the wavelength of the laser light. In order for the instrument to distinguish between forward-moving and backward moving surfaces, an acousto-optic modulator introduces constant frequency shift of $40 \mathrm{MHz}$. With no vibrations present, the receiver registers the carrier frequency of $40 \mathrm{MHz}$. In the presence of vibration, the Doppler-shifted signal frequency modulates the $40 \mathrm{MHz}$ carrier. The signal processor demodulates the signal, thereby retrieving the vibrational information.

LDV offers important advantages over other methods for measurement of BM motion. It does not require lowering the perilymph level as is necessary for the capacitive probe (Wilson, 1973; Wilson and Johnstone, 1975; Le Page, 1986) nor does it require a gamma radiation source, with attendant toxicity and mass loading problems associated with the Mössbauer technique (Sellick et al., 1982; Klianga and Khanna, 1983; Rhode 1971). Perhaps the most important advantages of LDV are its specified linearity of $1.0 \%$, dynamic range ( $>160 \mathrm{~dB}$ ), bandwidth $(0.1$ to $1 \mathrm{MHz}$ ), and its ability to make small (in diameter) point measurements.

Two factors make LDV measurements practical. First, it can be readily adapted to a compound microscope configured for vertical illumination. That is, the laser beam is directed through the viewing objective lens. Second, high refractive in$\operatorname{dex}(\mu=2.1)$, solid glass beads with diameters as small as a $10 \mu \mathrm{m}$ can be placed on the BM to serve as reflective objects.

We have begun using an implementation of LDV and obtained measurements of basilar membrane motion with little difficulty. Displacement input/output intensity functions showed compressive nonlinear growth for the topographic best frequency and the frequency tuning curves (isodisplacement functions) had sharply tuned tip regions.

\section{Materials and Methods}

\section{LDV-Microscope design}

The implementation of apparatus described here is straightforward and was used to obtain the data presented below. We consider it to be a first generation system being capable of only single point measurements, not differential measurements. The principles used, however, are quite general as is evident from comparing this design with that in the companion paper by Ruggero and Rich (1990) where single point measurements were accomplished using an LDV from a different manufacturer.

Fig. 1 gives a schematic drawing of the LDVmicroscope. The laser beam of the LDV is treated like the illumination source of any epi-illumination microscope. That is, the beam is directed through the objective lens. The system uses a beam splitter which serves to direct the beam into the image pathway instead of a fluorescence filter set (as would be used for epifluorescent microscopy). This arrangement is often referred to as vertical illumination and the use of a beam splitter is common in materials science for surface studies of specimens. In practice, it does not matter whether the beam comes into the image path via the 'vertical illumination port' or via the 'trinocular port' of a microscope.

Since the LDV analyzes the returned, Doppler-shifted light, the nature of the beam splitter can be important in some applications. It is a source of light loss, as each time the beam passes through (first the outgoing beam and then the reflected beam) some intensity is lost. If necessary the beam splitter could be replaced with a mirror (or partial mirror) sacrificing some or all the ability to visualize the specimen.

Fig. 1 shows only two focusing elements, the objective lens $\left(\mathrm{L}_{1}\right)$ and a lens $\left(\mathrm{L}_{2}\right)$ near the laser light source. The choice of an infinity corrected microscope objective lens simplifies the remaining optical design considerably. With an infinity corrected lens, parallel light rays are brought into focus so that, in effect, $L_{2}$ is a collimating lens. If the laser light is directed through an optical fiber (light guide), as in the case of the Polytec LDV, an image of the fiber (diameter) is transferred to the object plane, reduced in size by the ratio of the focal length of $L_{1}$ to the collimating lens, $L_{2}$. Since the diameter of the Polytec light guide is $5 \mu \mathrm{m}$, it is easy to achieve a diffraction limited spot size (i.e. determined by the numerical aperture of the objective lens). Beam expansion lenses may also 
be used to control the actual size of the focused spot in the object plane when using a laser beam directly.

The remaining optical element of importance in Fig. 1 is the filter in the visual pathway. Obviously the reflected beam can be very bright and should not be directly viewed by the eye. This filter can be a simple neutral density filter of at least 3 or 4 density, or short wavelength-pass interference filter. Some compromise is required to visualize the position of the laser beam when it is not returned by a reflector because the intensity of reflected light is very low from biological tissue.

The current work used the Polytec OFV-1000 fiber type laser vibrometer. In this instrument, both the outgoing laser light and the returned Doppler shifted light are handled through one polarization preserving optical fiber. As mentioned above, the light is kept separate in the two directions by cross polarization. An advantage of this fiber system that is potentially useful to electrophysiological experiments is that all of the electrical components of the system are in one chassis. Only a light guide needs to be brought into the sound booth/Faraday cage. A disadvantage of the fiber system is a sacrifice in sensitivity to detect and analyze light returned from low reflectance objects.

\section{Animal preparation}

Pigmented guinea pigs were pre-anesthetized with nembutal $(15 \mathrm{mg} / \mathrm{kg}, \mathrm{IP})$ and anesthetized with Innovar-Vet $(0.4 \mathrm{ml} / \mathrm{kg}$, IM). Supplemental doses of Innovar-Vet were given hourly to maintain an acceptable surgical anesthesia level $(0.2$ $\mathrm{ml} / \mathrm{kg} \mathrm{IM}$ ). The general physiological condition of the animal was monitored by recording the heart rate and the body temperature. A tracheostomy and tracheal canulation maintained the airway although artificial ventilation was not used. The

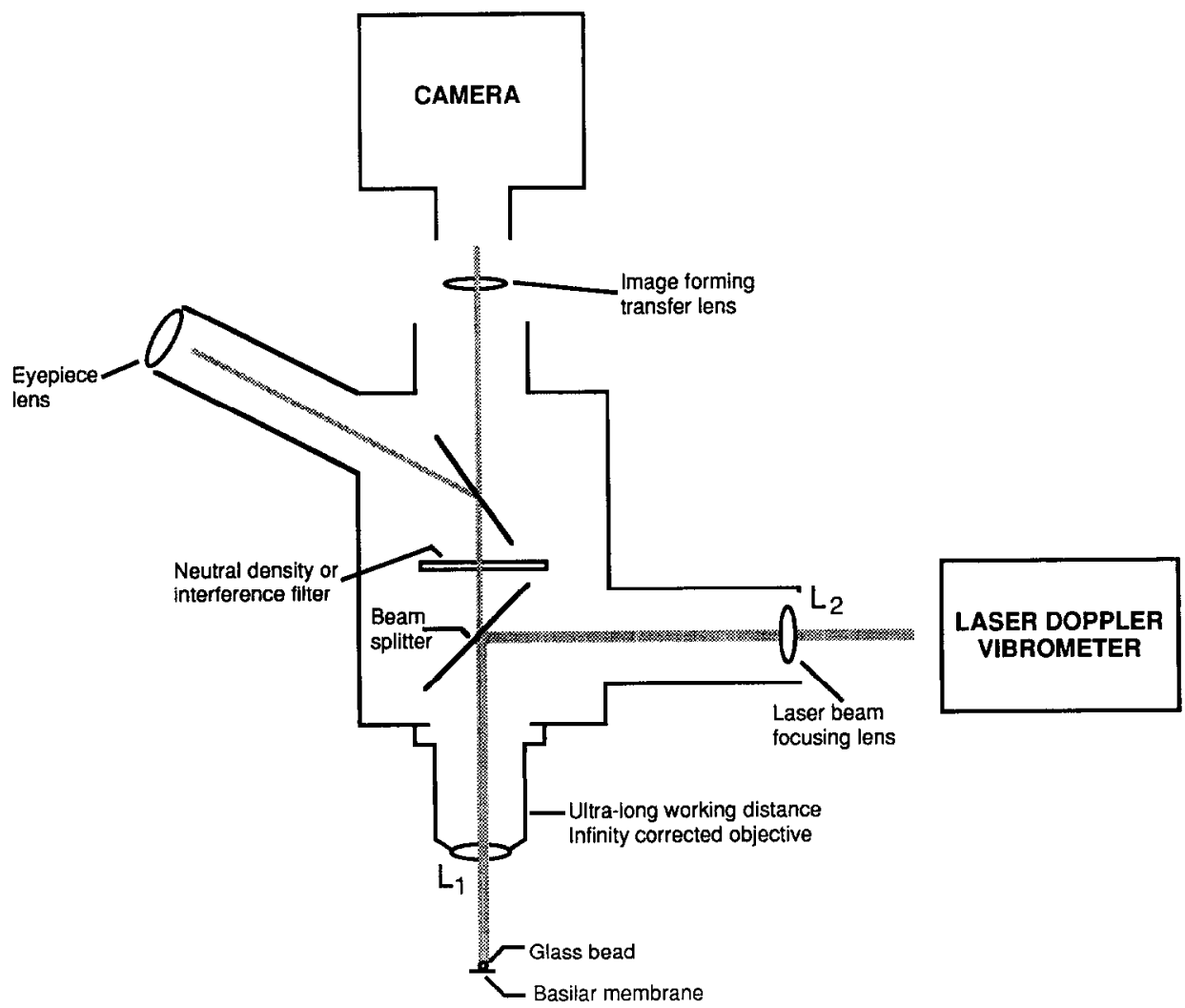

Fig. 1. A schematic diagram of the microscope system. The laser Doppler vibrometer is coupled to the microscope using a collimating lens $\left(\mathrm{L}_{2}\right)$ and a beam splitter. Measurements are made from reflecting objects placed on the vibrating surface (glass beads). 
head was fastened into a heated head holder (Brown et al., 1983; Shore and Nuttall 1985) and a ventral and postauricular surgical dissection exposed the bulla which was carefully opened over a wide area. Although the tympanic membrane and ossicles were preserved, the tensor tympani muscle tendon was transected.

The cochlea was opened by carefully thinning the bony wall over the scala tympani of the first (basal) turn. This was accomplished using a narrow scalpel blade in a technique fully described by Brown and Nuttall (1984). The opening in the cochlea was about $0.5 \mathrm{~mm}$ in diameter and only the amount of perilymph necessary to clear and control bleeding was removed. The perilymph level returned to fill the cochlea by natural flow of cerebrospinal fluid. The head was oriented so that basilar membrane was approximately horizontal in the region directly adjacent to the opening. Thus the microscope which views the basilar membrane was in the vertical position.

An ear speculum fitted into the external meatus delivered sound in a closed field manner. The sound source was a $1 / 2$ inch Bruel and Kjaer condenser microphone. Sound levels were calibrated using a probe microphone system ( $1 \mathrm{~mm}$ probe tube $\mathrm{B}$ and $\mathrm{K} 1 / 2$ inch condenser microphone) placed within $2 \mathrm{~mm}$ of the tympanic membrane. A silver wire electrode was fixed into position on the round window membrane to record the cochlear compound action potentials (CAP). The reference electrode was a chlorided silver wire placed in the neck musculature. Tone bursts $(1 \mathrm{~ms}$ rise $/$ fall time, $10 \mathrm{~ms}$ duration) given $10 / \mathrm{s}$ at frequencies between 1 and $40 \mathrm{kHz}$ served to derive a CAP audiogram monitoring the condition of the cochlea.

\section{Reflective microbeads-Design considerations}

In the current work, glass microspheres were selected to act as light reflecting objects. The solid glass microbeads (Mo-Sci Corp., Rolla, MO) of $10-30 \mu \mathrm{m}$ diameter and refractive index 2.1 were coated with poly-L-lysine to enhance their adhesion to the BM. A small number of beads were cleaned by placing them in a test tube containing $1 \mathrm{ml}$ of $100 \% \mathrm{EtOH}$. Following agitation and centrifugation, the EtOH supernate was pipetted off and the beads were rinsed three times with distilled water $\left(\mathrm{dH}_{2} \mathrm{O}\right)$ following the same sequence of agitation, centrifugation, and removal of supernate. Following the third rinse, poly-Llysine (Sigma Diagnostics) $0.01 \% \mathrm{w} / \mathrm{v}$ in $\mathrm{dH}_{2} \mathrm{O}$ $(0.5 \mu \mathrm{M})$ was placed in the test tube for a period of 5 min during which the test tube was agitated periodically. The supernate was removed, the beads were rinsed three times with $0.9 \%$ sterile saline, and were maintained in saline until use. The internal surface of a micropipette was also coated with poly-L-lysine, rinsed three times with and filled with physiologic saline. A small quantity of microbeads was taken by suction into the filled micropipette. The pipette was held in a micromanipulator and positioned through the opening in the cochlea. Beads were dispensed into the cochlea by gently forcing them from the pipette. They fell by gravity onto the BM.

The microbeads were readily visible with a dissecting microscope so that one could ascertain whether they were located in a position that would provide useful information about vibration of the BM. The compound microscope with LDV was then positioned over the cochlea and the microbeads on the basilar membrane were brought into focus. It is merely necessary to move the position of the head (or microscope) to position the laser beam onto a microbead. Alternatively, the beam itself might have been steered.

One can expect the reflected light from a bead to result either from total internal reflection in the glass bead or from a surface reflection. Fig. 2 a illustrates the first case where it is assumed that total internal reflection accounts for the reflected light. Since there is a layer of perilymph $(\mu=1.33)$ above the glass bead, the critical angle for the total internal reflection to occur may be calculated from Snell's law * to be 39.3 degrees. The angles are computed with respect to the normal to the surface. Then the angle made by the back refracted light would be 57.4 degrees as also obtained from Snell's law. But since the numerical aperture of the objective is only 0.4 , which is equivalent to an angle of 23.6 degrees, the returned light energy due to the total internal reflec-

* See a detailed explanation of these laws in any standard optical physics text book, e.g., Rudolf Kingslake, Optical System Design, Academic Press, Orlando, Florida (1983). 
tion would miss the objective (Olympus, $20 \times$ 1/0.4 NA). Therefore almost all the reflected light is probably directly from the surfaces of the microbead.

From geometrical optics, one can show that the back scattered light must fall inside a cone of 35 degrees as illustrated in Fig. 2b. From Fresnel's laws *, of reflection off a transparent surface, the reflected energy can be computed as $4.5 \%$ of the incident energy. Hence for the optical performance of the LDV, reflections have to occur from both the top and the bottom surfaces of the glass bead. To achieve this, $\mathrm{L}_{2}$ must collimate the laser light on $\mathrm{L}_{1}$ to a beam diameter less than $4.8 \mathrm{~mm}$. Then $L_{1}$, in turn, will focus the light to a small spot on the top surface of the glass bead. About $4.5 \%$ of the light energy is reflected off the top surface and $95.5 \%$ passes through under optimal conditions mentioned above. Again, about $4.5 \%$ of this light energy is reflected by the bottom surface and about $4.1 \%$ of the initial light energy reaches back $\mathrm{L}_{1}$. Thus, approximately about $8.6 \%$ of the incident light energy is reflected back and a portion of this reaches LDV depending upon the light guide efficiency. Note that this analysis is intended to provide the reader a rough estimate of
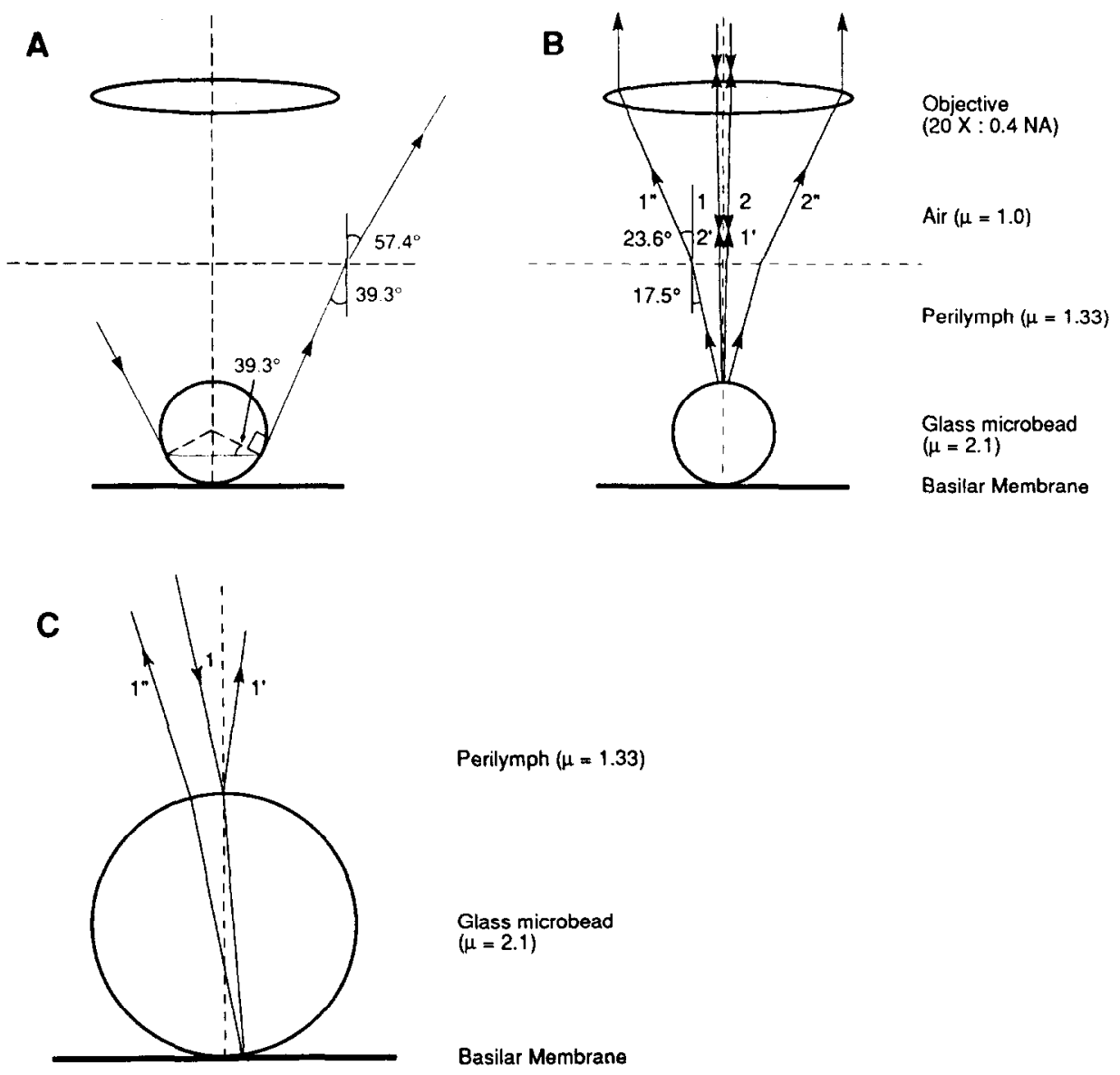

Fig. 2. Light ray diagrams showing the refraction of the laser light does not account for backscattered energy. A) A light ray which undergoes total internal reflection in the glass bead will have an angle of 39.3 degrees from the perpendicular. The ray will experience a further deflection as it crosses the air-perilymph boundary. A microscope objective of 0.4 NA will not accept a light ray having such a large angle. B) Geometric optical considerations predict that a ray 1 gets reflected off the top and the bottom surfaces (of the glass bead) as ray $1^{\prime}$ and ray $1^{\prime \prime}$. If the light rays 1 and 2 form the extreme rays of a light beam falling on the objective, the beam width must be smaller than $4.8 \mathrm{~mm}$ for the maximum returned light. C) An enlarged schematic of the glass microbead. Only paths of incident ray 1 and the reflected rays $1^{\prime}$ and $1^{\prime \prime}$ are shown for clarity. 
the reflections of laser off the glass bead and is based on geometrical optics. For the sake of simplicity of analysis, the physical apertures of $\mathrm{L}_{1}$ and $L_{2}$ are assumed to be the same. This above arrangement is also potentially important for the following reasons: It allows use of a low power laser (the Polytec laser is less than $1 \mathrm{~mW}$ ). A small spot size also allows a large number of smaller beads on the BM for multiple point measurements.

Signals from the LDV were typically too small to be seen without some signal processing. While the time waveforms can be readily derived by signal averaging, this study used a lock-in amplifier (Stanford model SR530) to derive the magnitude and phase of BM motion for pure tone signals.

Sounds were delivered to the ear from a voltage controlled oscillator, with the aid of computer controlled attenuators and tone switches. A PC-AT type microcomputer was used to control the experiment and store the digitized data.

\section{Results}

Performance of the LDV-microscope system was characterized using a piezoelectric vibrator. Observations were made from glass microbeads on the vibrator and on the basilar membrane. It is possible to obtain sufficiently strong reflections from opaque objects, however biological tissues have relatively low reflectance and do not normally provide reflections suitable for measurement (Khanna et al., 1989). Therefore in practice useable signal cannot be obtained from the BM or the osseous spiral lamina. Microbeads provided sufficient reflected signal to give full scale readings in the signal strength meter of the LDV. Although the full scale signal would allow optimal performance of the instrument, weaker signals also give useable results.

Using the piezovibrater, (Spectra Corp., Rochester NY, Model 560) a strong reflected signal gave an effective noise floor of $1 \mu \mathrm{m} / \mathrm{s} \mathrm{rms}$ at frequencies between $1 \mathrm{kHz}$ and $30 \mathrm{kHz}$ when using a lock-in analysis time constant of 1 second. Even the weakest signal, a minimum reading on the Polytec signal strength meter, gave measurements that were only ten times less sensitive (i.e.

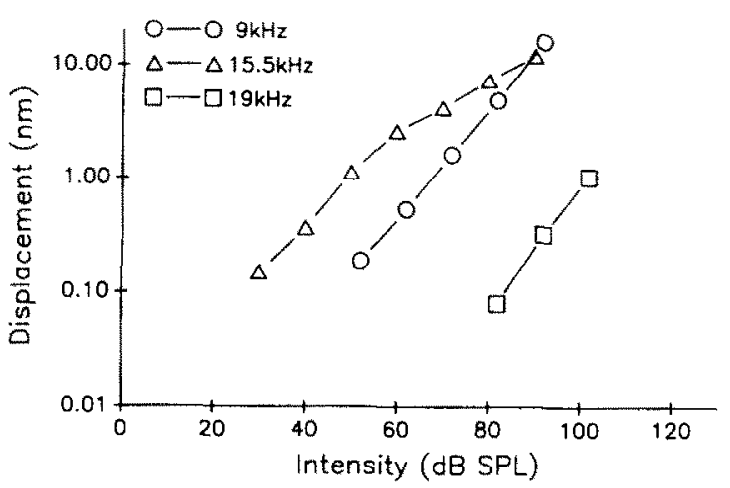

Fig. 3. Intensity (displacement input/output) functions obtained from a microbead located on the basilar membrane of the guinea pig. The characteristic frequency of the location was about $15.5 \mathrm{kHz}$ and the intensity function for this frequency of sound stimulation exhibits some nonlinear (compressive) growth.

$10 \mu \mathrm{m} / \mathrm{s}$ ). These velocities correspond to rms displacements at $20 \mathrm{kHz}$ of 8 and $80 \mathrm{pm}$ respectively. When measuring from a microbead on the BM the 'practical noise floor' of the system at high signal strength was an order of magnitude worse than the ideal. That is, the signal contained additional noise, perhaps from degraded optical properties of the system (the beads being under perilymph for example), and certainly from general movement of the animal. Thus the realistic minimum velocity recorded by a lock-in amplifier using a 1 s time constant is about $10 \mu \mathrm{m} / \mathrm{s}$. This level is less than the anticipated threshold of motion for the basilar membrane (Patuzzi et al., 1983)

Fig. 3 shows displacement intensity functions obtained from a microbead located on the basilar membrane at a distance of approximately $10 \mu \mathrm{m}$ radially from the osseous spiral lamina. These functions demonstrate the expected characteristics observed in previous studies from hair cell receptor potentials (Russell and Sellick, 1978; Goodman et al., 1982; Brown et al., 1983; Dallos, 1985). The dc receptor potential shows compressive nonlinear frequency dependent behavior. Near CF the growth of the receptor potential begins to saturate at lower sound pressure levels. Below CF there is a more linear (straight-line) relationship between receptor potential magnitude and sound pressure level. Similar results have been observed in the rate-level functions obtained from auditory nerve fibers (Kiang et al., 1965; Geisler et al., 1974; 
Sachs and Abbas, 1974; Evans, 1979). The compressive type nonlinear growth pattern seen for the CF (Fig. 3, $15.5 \mathrm{kHz}$ ) and the relatively linear (straight line) growth function for frequencies below CF has also been reported from other studies of basilar membrane motion (Rhode, 1971; Sellick et al., 1982). The shape of the intensity function near CF is dependent on physiological condition of the animal. Loss of neural threshold is associated with linearization of basilar membrane motion and loss of sensitivity (Sellick et al., 1982). Fig. $4 \mathrm{~b}$ shows a similar result in the basilar membrane displacement before and after death of the animal. There is approximately a $40 \mathrm{~dB}$ reduction in sensitivity and the slope of the function is steeper post mortem.

Examples of frequency tuning curves (FTCs) from two guinea pigs are shown in Fig. $5 a$ and $b$. In Fig. 5a a constant displacement of $0.5 \mathrm{~nm}$ is used, in $5 \mathrm{~b}$ the $0.5 \mathrm{~nm}$ criterion is compared to a constant velocity of $50.0 \mu / \mathrm{s}$ in the same animal. The forms of the tuning curves are comparable to FTCs from auditory nerve fibers (Kiang et al, 1965), inner hair cell receptor potential FTCs (Russell and Sellick, 1977, 1978; Goodman et al., 1982; Brown et al., 1983) and basilar membrane displacement (Khanna and Leonard, 1982; Sellick et al., 1983).

\section{Discussion}

The LDV is a relatively new commercial instrument that measures the vector component of

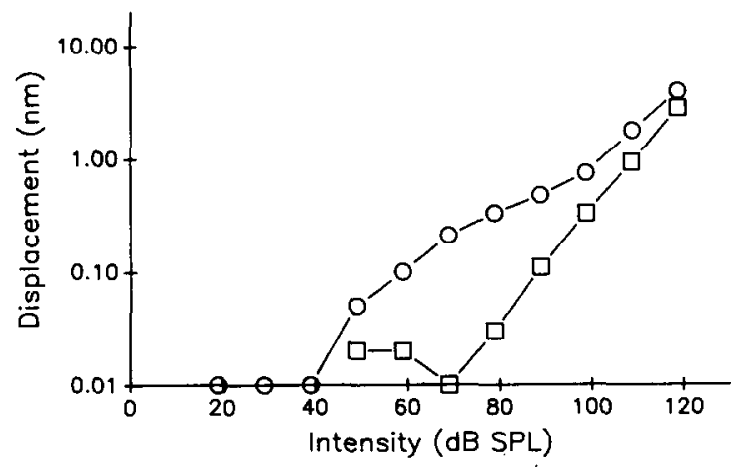

Fig. 4. A comparison of the intensity function obtained of the basilar membrane motion (at the characteristic frequency location) in the normal condition ( $\mathrm{O}-\mathrm{O}$ ) to that derived 30 minutes following death of the animal ( $\square-\square)$. The shift to the right which occurs is characteristic of many pathological processes which reduce cochlear sensitivity.

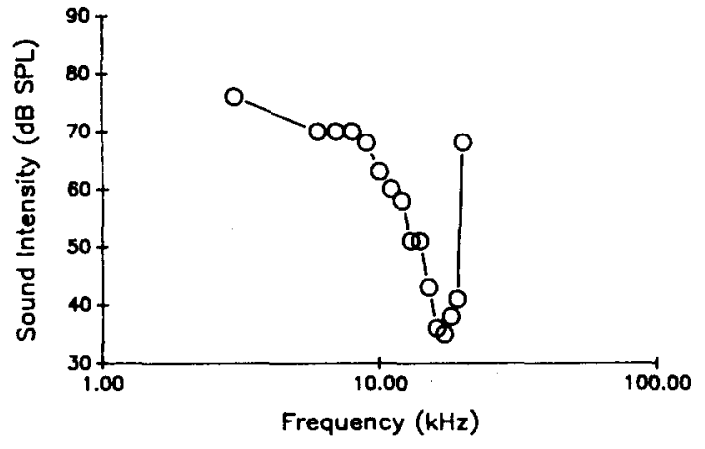

B

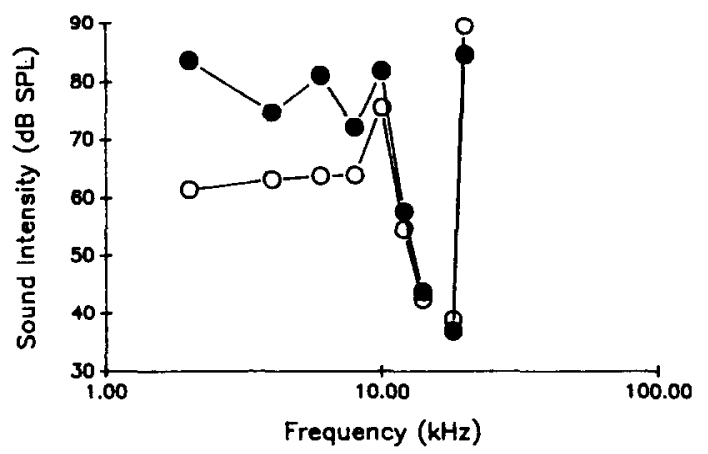

Fig. 5. Example frequency tuning curves from two different guinea pigs. Iso-displacement functions $(0.5 \mathrm{~nm})$ are given by the open symbols $(\mathrm{O}-\mathrm{O})$. In panel $\mathrm{B}$ the iso-displacement function is compared with an iso-velocity curve (50 $\mu \mathrm{m} / \mathrm{s})(-)$ ) interpolated from the same data.

velocity directed along the axis of the laser beam. Related instrumentation uses multiple beams to determine the velocity of objects crossing the beams (laser anemometers). The velocity recorded is an instantaneous time measurement and is directionally sensitive. This is accomplished by heterodyne techniques and frequency demodulation electronics.

Laser Doppler measurements of moving objects observed with a microscope has previously been reported, most often in the study of the velocity of red blood cells flowing in capillaries (eg Johnson, 1984; Born et al., 1978; Einav et al., 1975). Regarding BM motion, both homodyne and heterodyne interferomic techniques have been used in combination with microscopy by Khanna and colleagues (eg. Khanna, 1986; Willemin et al., 1988). In these studies, however, the interferometry technique was a custom application and was not easily duplicated by other laboratories. In some studies 
involving interferometry technique, the reflective object placed on the basilar membrane was a flat mirror (eg. a gold foil) and potentially caused damage to the organ of Corti (Leonard and Khanna 1984).

\section{Comparison of $L D V$ to other techniques}

Measurement of basilar membrane motion has been accomplished using many different methods. In order of appearance in the literature they are direct optical microscopic observations (Bekesy, 1960), capacitive probe (Wilson and Johnstone, 1975), Mössbauer velocimetry (Johnstone and Boyle, 1967) laser speckle detection (Kohlloffel, 1972) homodyne interferometry (Khanna, 1982), optical lever (La Page, 1988), heterodyne interferometry (Khanna et al., 1989), and Doppler vibrometry (Nuttall et al., 1989). It is probably valid to say that only Mössbauer technique, heterodyne interferometry, and the optical lever are viable contemporary choices for study of organ of Corti motion. With the current demonstration of LDV feasibility, most of the rationale for use of the older, non laser based methods is rendered moot. One potential remaining advantage of the Mössbauer technique, however, is the ability to measure the velocity vector in any direction as some of the gamma radiation may pass through the bone and tissue of the head. LDV in contrast requires an unimpeded optical path of the laser illumination to the organ of Corti. Significant contamination of the perilymph (by blood for example) can scatter the light reducing the Doppler shifted signal.

Willimen et al., 1989 has discussed the relative merits of heterodyne interferometery over homodyne techniques. With sufficient sensitivity for processing the reflected Doppler shifted signal it would be possible to record the movement of the basilar membrane without the aid of reflective objects. Indeed laser interferomic techniques can be combined with the ability of the high numerical aperture lens and analog optical methods, such as confocal techniques, to measure the motion of in-focus thin optical sections of the organ of Corti (Khanna and Koester, 1989). At the time of the current report we have not had the opportunity to explore the lower limit of reflectance usable by the Polytec LDV but it is conceivable that useful signal levels could be obtained from some portions of the organ of Corti and for high sound stimulation levels where velocities would be greater. In practice, the LDV method is limited to the ability to place a reflective object on surface of interest. We have used glass spheres but higher reflectance spheres of smaller size could be used to seed other areas of the organ of Corti than the basilar membrane. Obviously some creative methodological developments in this regard would open some exciting new directions in the physiology of organ of Corti micromechanics.

Ruggero and Rich (1990) present a thorough comparison of Mössbauer technique and LDV. It is clear that LDV exceeds the sensitivity of the Mössbauer technique for absolute velocity determinations and is not subject to such a nonlinear and limited dynamic range as Mössbauer technique. The exact performance of $\mathrm{LDV}$, however depends on the success of a manufacturer to implement an efficient engineering design. At the time of this manuscript preparation a full characterization of instrument performance (and comparison among manufacturers) has not been made but it is clear that the manufacturers make significantly different claims for performance specifications.

An important contemporary question of cochlear physiology is the slow position changes of the basilar membrane that may accompany the vibration pattern of the acoustic stimulus. Both capacative probe technique (LaPage, 1987) and the optical lever (LaPage, 1988) have been used to study the 'dc position' of the basilar membrane. These methods are well suited to the task of measuring steady state position of an object placed near the probe. LDV offers an opportunity to explore the dc-position of the BM by way of two approaches. In the first approach one can choose to analyze any frequency component of the Doppler (velocity) signal. The linearity of the instrument is ideal for distortion product measurements. Obviously there is no velocity component for constant dc shifts of the BM, however modulated carriers offer a way to explore the phenomenon. Using a lock-in amplifier it is feasible to accurately measurc frequency components less than $1 \mathrm{~Hz}$. For arbitrarily small carrier modulation frequencies this becomes an approximation of the dc shift. We 
have reported preliminary data that energy may be present for carrier frequencies below $10 \mathrm{~Hz}$ corresponding to rms displacements up to $1 \mu \mathrm{m}$ (Nuttall et al., 1989, 1990).

The second approach can actually measure the dc position shifts. This method uses a fringe counter to measure absolute position changes. Current implementations of fringe counters in LDV's can achieve displacement resolutions of up to $1 / 100$ of a wavelength of $\mathrm{He}-\mathrm{Ne}$ laser light. This corresponds to $6.3 \mathrm{~nm}$. Our preliminary experience using fringe counting to detect BM motion was disappointing as general animal motion caused an overwhelming baseline noise level. The problem of animal motion may be solved in future work by signal averaging techniques and most importantly by differential measurements comparing $\mathrm{BM}$ motion to osseous spiral lamina motion for example.

In the current study, we have shown that the commercial LDV is reliable and easily attached to a conventional (infinity corrected) compound microscope. The use of glass microbeads on the basilar membrane solves some previous problems of both the Mössbauer and the laser experiments. First the glass sphere is of very low mass. A $20 \mu \mathrm{m}$ diameter solid glass sphere of refractive index 2.1 may be calculated to have a mass of $11.7 \mathrm{ng}$ assuming a density of $2.8 \mathrm{~g} / \mathrm{cc}$. This is considerably smaller than typical Mössbauer sources $(5 \times$ $40 \times 40 \mu \mathrm{m}$ and $0.1 \mu \mathrm{g}$, Rhode and Robles 1974) and similar to the gold foil reflector (50-120 $\mu \mathrm{m}$ diameter and $10 \mathrm{ng}$, Khanna, 1982). The sphere is an omnidirectional reflector so that orientation on the BM becomes unimportant. The microsphere size governs its ability to specify a point measurement on the BM and diameters less than $5 \mu \mathrm{m}$ are obtainable. The mass of a $5 \mu \mathrm{m}$ diameter bead is $0.18 \mathrm{ng}$. Obviously multiple measurements of BM motion based on position become feasible because many small low mass beads can be scattered onto the BM.

Useful reflected signal levels may theoretically be gained by increasing the power of the laser illumination. However there is a potentially toxic effect of light on the physiology of the organ of Corti. Willemin et al. (1988) suggest the guideline proposed for the retina $\left(0.5 \mathrm{~W} / \mathrm{cm}^{2}\right)$ which is equivalent to the power density of a $0.5 \mathrm{~mW}$ laser beam of about $1 \mathrm{~mm}$ diameter. In practice Wil- lemin et al. (1989) and Khanna and Koester (1989) describe the focusing of a $0.9 \mathrm{~mW} \mathrm{He}-\mathrm{Ne}$ laser to a spot size of $5 \mu \mathrm{m}$ in the object plane. Thus energy densities employed in the contemporary laser based organ of Corti vibration studies are similar. The question of what is the maximum power level to be used while retaining normal physiology remains to be explored. Although wavelength of illumination (i.e. absorptance) is an important caveat, Lin and Nuttall (1990) have found that isolated outer hair cells tolerated approximately $5 \times 10^{3} \mathrm{~W} / \mathrm{m}^{2}$ of blue light for at least $30 \mathrm{~min}$ when the cells were not stained by various dyes which tended to enhance phototoxicity.

It should be noted that the microsphere reflector is theoretically not required. Khanna and Koester (1989) have achieved this in practice using a more efficient custom interferometer. Biological tissue, however, has very low reflectance (Khanna et al., 1989) no useful signal has been obtained in our work without the reflective object on the BM.

The intensity functions and tuning curves shown in this report demonstrate the feasibility of the LDV system. We consider these data to be early results and subject to some degradation based on surgical preparation difficulty. We believe that further refinements in both experimental sophistication and physiological quality will yield a large amount of new and detailed information on physiology and mechanics of organ of Corti.

\section{Acknowledgements}

This work was supported by NIH research grant NS 15107. The authors thank the Polytec Corp (Redwood, CA) for the two week loan of the OVF-1000 during which time this study was accomplished and the Mo-Si Corp. for the gift of glass microbeads.

\section{References}

Békésy, G. von. (1960) Experiments in Hearing. (McGraw Hill, NY).

Born, G.V., Melling, A. and Whitelaw, J.H. (1978) Laser Doppler microscope for blood velocity measurements. Biorheology 15, 163-172.

Brown, M.C., Nuttall, A.L. and Masta, R.I. (1983) Intracellular recordings from cochlear inner hair cells: Effects of 
stimulation of the crossed olivocochlear efferents. Science 222, 69-72.

Brown, M.C., Smith, D.I. and Nuttall, A.L. (1983) Anesthesia and surgical trauma: Their influence on the guinea pig compound action potential. Hear. Res. 10, 345-358.

Brown, M.C. and Nuttall, A.L. (1984) Efferent control of cochlear inner hair cell response in the guinea pig. $\mathrm{J}$. Physiol. 354, 625-646.

Dallos, P. (1985) Response characteristics of mammalian cochlear hair cells. J. Neurosci. 5: 1591-1608.

Durrani, T.S. and Greated, C.A. (1977) Laser Systems in Flow Measurement, Plenum Press, New York.

Einav, S., Berman, H.J., Fuhro, R.L., DiGiovanni, P.R. Fridman, J.D. and Fine, S. (1975) Measurement of blood flow in vivo by laser Doppler anemometry through a microscope. Biorheology 12, 203-205.

Evans, E.F. (1979) Single unit studies of the mammalian auditory nerves. In: H.A. Beagley (Ed.) Auditory Investigations: The Oxford Univ. Press, U.K.

Geisler, C.D., Rhode, W.S. and Kennedy, D:T. (1974): Responses to tonal stimuli of single auditory nerve fibers and their relation to basilar membrane motion in the squirrel monkey. J. Neurophysiol. 37, 1156-1172.

Goodman, D.A., Smith, R.L. and Chamberlain, S.C. (1982): Intracellular and extracellular responses in the organ of Corti of the gerbil. Hear. Res. 7, 161-179.

Johnson, R.P. Laser Doppler microscopy. (1978) Biochem Soc Trans 12, 635-637.

Johnstone, B.M. and Boyle, A.J.F. (1967) Basilar membrane vibration examined with the Mössbauer technique. Science 158, 389-390.

Khanna, S.M. (1986) Homodyne interferometer for basilar membrane measurements. Hear. Res. 23, 9-26.

Khanna, S.M. and Koester, C.J. (1989) Optical sectioning characteristics of the heterodyne interferometer. Acta Otolaryngol (Stockh.) Suppl. 467, 61-68.

Khanna, S.M., Flock, A. and Ulfendahl, M. (1989) Comparison of the tuning of outer hair cells and the basilar membrane in the isolated cochlea. Acta Otolaryngol (Stockh.) Suppl. 467, 151-156.

Khanna, S.M., Johnson, G.W. and Jacobs, J. (1986) Homodyne interferometer for basilar membrane measurements. II. Hardware and techniques. Hear. Res. 23, 27-36.

Khanna, S.M. and Leonard, D.G.B. (1982) Basilar membrane tuning in the cat cochlear. Science 215, 305-306.

Khanna, S.M., Willemin, J.F. and Ulfendahl, M. (1989) Measurements of optical reflectivity in cells of the inner ear. Acta Otolaryngol (Stockh.) Suppl. 467, 69-75.

Kiang. N.Y.S., Watanabe, T., Thomas, E.C. and Clark, L.F. (1965) Discharge patterns of single fibers in the cat's auditory nerve. Research Monograph No. 35 (MIT, Cambridge).

Kliauga, P. and Khanna, S.M. (1983) Dose rate to the inner ear during Mössbauer experiments. Phys. Med. Bio. 28, 359366.

Kohllöffel, L.U.E. (1972a) A study of basilar membrane vibrations. I. Fuzziness-detection: A new method for the analysis of microvibrations with laser light. Acustica 27, 49-65.

Kohllöffel, L.U.E. (1972b) A study of basilar membrane vibra- tions. II. The vibratory amplitude and phase pattern along the basilar membrane (post-mortem). Acustica 27, 66-81.

Kohllöffel, L.U.E. (1972c) A study of basilar membrane vibrations. III. The basilar membrane frequency response curve in the living guinea pig. Acustica 27, 82-89.

Leonard, D.G.B. and Khanna, S.M. (1984) Histological evaluation of damage in cat cochleas used for measurement of basilar membrane mechanics. J. Acoust Soc. Am. 75: 515527.

LePage, E.L. (1987) Frequency-dependent self-induced bias of the basilar membrane and its potential for controlling sensitivity and tuning in the mammallian cochlea. J. Acoust. Soc. Am. 82, 139-154.

LePage, E.L. (1987) The application of a capacitative probe technique for direct observation of electromechanical processes in the guinea pig cochlea. J. Acoust. Soc. Am. 82, 126-138.

LePage, E.L. (1988) Fiber optic lever reveals sound-produced contractile component of basilar membrane motion. Abstr. Assoc. Res. Otolaryngol. Clearwater Beach, FL.

Lin, X. and Nuttall, A.L. (1990) An evaluation of the parameters of voltage sensitive dyes on outer hair cells stained in vitro. Abstr. Assoc. Res. Otolaryngol. Clearwater Beach, FL.

Nuttall, A.L., Dolan, D.F. and Avinash, G. (1989) Laser Doppler vibrometer measurements of basilar membrane motion in the guinea pig. Soc. Neurosci. Meet. Phoenix. AZ.

Nuttall, A.L., Dolan, D.F. and Avinash, G. (1990) Laser Doppler vibrometry: A new method for basilar membrane vibration measurement. Abstr. Assoc. Otolaryngol. Clearwater Beach, FL.

Patuzzi, R.B. and Sellick, P. (1983) A comparison between basilar membrane and inner hair cells receptor potential input-output functions in the guinea pig cochlea. J. Acoust. Soc. Am. 74, 1731-1741.

Rhode, W.S. and Robles, L. (1974) Evidence from Mössbauer experiments for nonlinear vibrations in the cochlea. $J$. Acoust. Soc. Am. 55, 588-596.

Rhode, W.S. (1971) Observations of the vibration of the basilar membrane in squirrel monkeys using the Mössbauer technique. J. Acoust. Soc. Am. 49, 1218.

Ruggero, M.A. and Rich, N.C. (1991) Application of a commercially-manufactured Doppler-shift laser velocimeter to the measurement of basilar-membrane vitration. Hear. Res. 51 , (in press).

Russell, I.J. and Sellick, P.M. (1978) Intracellular studies of hair cells in the mammalian cochlea. J. Physiol. 284, $261-$ 290.

Russell, I.J. and Sellick, P.M. (1977) Tuning properties of cochlear hair cells. Nature 267, 858-860.

Sachs, M.B. and Abbas, P.J. (1974) Rate versus level functions for auditory-nerve fibers in cats: Tone burst stimuli J. Acoust. Soc. Am. 56, 1835-1847.

Sellick, P.M., Patuzzi, R. and Johnstone, B.M. (1982) Measurement of basilar membrane motion in the guinea pig using the Mössbauer technique. J. Acoust. Soc. Am. 72, 131-141. Sellick, P.M., Patuzzi, R. and Johnstone, B.M. (1983) Compari- 
son between the tuning properties of inner hair cells and basilar membrane motion. Hear. Res. 10, 93-100.

Sellick, P.M., Yates, G.K. and Patuzzi, R. (1983) The influence of Mössbauer source size and position on phase and amplitude measurements of the guinea pig basilar membrane. Hear. Res. 10, 101-108.

Shore, S.E. and Nuttall, A.L. (1985) The effects of cochlear hypothermia on compound action potential tuning. $J$. Acoust. Soc. Am. 77, 590-598.
Willemin, J.-F., Dändliker, R. and Khanna, S.M. (1988) Heterodyne interferometer for submicroscopic vibration measurements in the inner ear. J. Acoust. Soc. Am. 83, 787-795.

Wilson, J.P., Johnstone, J.R. (1975) Basilar membrane and middle-ear vibration in guinea pig measured by capacitive probe. J. Acoust. Soc. Am. 57, 705-723.

Wilson, J.P. (1973) A sub-miniture capacitative probe for vibration measurements of the basilar membrane. J. Sound. Vib. $30,483-493$. 